Storia sacra e profana nei volgarizzamenti medioevali 


\section{Beihefte zur Zeitschrift für romanische Philologie}

Herausgegeben von

Claudia Polzin-Haumann und Wolfgang Schweickard Band 436 


\section{Storia sacra e profana nei volgarizzamenti medioevali}

Rilievi di lingua e di cultura

A cura di

Michele Colombo, Paolo Pellegrini

e Simone Pregnolato

\section{DE GRUYTER}


La pubblicazione di questo volume ha ricevuto nel 2019 il contributo finanziario dell'Università Cattolica del Sacro Cuore sulla base d'una valutazione dei risultati della ricerca in essa espressa e il contributo del Dottorato di Ricerca in Filologia, Letteratura e Scienze dello Spettacolo dell'Università degli Studi di Verona.

ISBN 978-3-11-060857-1

e-ISBN (PDF) 978-3-11-061111-3

e-ISBN (EPUB) 978-3-11-060864-9

ISSN 0084-5396

Library of Congress Control Number: 2019947689

\section{Bibliografische Information der Deutschen Nationalbibliothek}

Die Deutsche Nationalbibliothek verzeichnet diese Publikation in der Deutschen Nationalbibliografie; detaillierte bibliografische Daten sind im Internet über http://dnb.dnb.de abrufbar.

(C) 2019 Walter de Gruyter GmbH, Berlin/Boston

Satz: Integra Software Services Pvt. Ltd.

Druck und Bindung: $\mathrm{CPI}$ books $\mathrm{GmbH}$, Leck

www.degruyter.com 


\section{Sommario}

Prefazione - VII

\section{Storia sacra}

Nicolangelo D’Acunto

L'ascesi narrata. Varia fortuna delle Vite dei Padri del deserto fra imitazione monastica e penitenza istituzionalizzata -3

Raymund Wilhelm

Bonvesin da la Riva agiografo e volgarizzatore. Dagli exempla della Vita scholastica ai miracoli in volgare -19

Elisa De Roberto

Raccontare il miracolo nel Medioevo italiano. Aspetti pragmatici e testuali della letteratura miracolistica in volgare - 41

Massimo Zaggia

Alle origini della storia sacra: l'avvio del Genesi in volgare italiano -85

Paolo Pellegrini

«Sul cavoge» / «sui cavegi». Nota sul pronome enclitico in italiano antico — 149

Michele Colombo

Lettera e voce nella «Passione Mai» in veneziano antico 155

\section{Storia profana}

Roberta Cella

L'epistola dei palermitani ai messinesi (13 aprile 1282)

e il suo volgarizzamento — 173

Simona Brambilla

Note sul volgarizzamento della Fam. xII 2 di Francesco Petrarca -197

\section{Chiara De Caprio}

Figure dell'autore nei volgarizzamenti e nelle cronache in volgare. Aspetti teorici e linee di una ricerca storico-linguistica nei testi medioevali — 211 
VI - Sommario

\section{Enrico Faini}

Vegezio e Orosio: storia, cavalleria e politica nella Firenze

del tardo Duecento — 237

Cristiano Lorenzi

Tradurre la storia romana. Il caso delle due redazioni del volgarizzamento della prima Catilinaria fra Due- e Trecento — 255

Giulio Vaccaro

Storia e geografia di un centone di volgarizzamenti: il Libro dell'Aquila -273

Luca Barbieri

Materia troiana e materia ovidiana nel ms. Gaddi 71 della Biblioteca Laurenziana di Firenze - 299

Simone Pregnolato

La «verace ystoria». Avviamento allo studio del volgarizzamento troiano di Mazzeo Bellebuoni — 319

\section{Indici}

1 Indice dei nomi - 375

2 Indice dei manoscritti e dei documenti d'archivio - 381 


\title{
Lettera e voce nella «Passione Mai» in veneziano antico
}

\begin{abstract}
The «Passione Mai» is a Passion narrative which harmonizes the four Gospels. The text is preserved in a fourteenth-century manuscript of the Angelo Mai Library in Bergamo (Italy), and its prologue clearly shows that it was intended for reading aloud on Good Friday, perhaps in a brotherhood of laymen. The study argues that the «Passione Mai» is a translation from Latin and analyses its language, identifying it as ancient Venetian. Nonetheless, the language shows a few non-Venetian features, together with a Latin influence.
\end{abstract}

Keywords: Gospel harmonies; vernacular translations of the Bible; ancient Venetian; reading aloud

\section{Scrittura e oralità nel Basso Medioevo}

Nel 1987, il filologo e critico letterario ginevrino Paul Zumthor pubblicò un libro intitolato La lettre et la voix: de la «littérature» médiévale, affermatosi come un caposaldo negli studi letterari sul Medioevo. La sua tesi centrale è che «l'insieme dei testi che ci sono pervenuti del X, XI, XII secolo e, in misura forse minore, del XIII e del XIV, è passato attraverso la voce non in modo casuale, ma in virtù di una situazione storica che ha fatto di questo transito vocale l'unico modo possibile di realizzazione - di socializzazione - di questi testi» (Zumthor 1990, 29). L'assunto è argomentato elencando gli indizi di oralità presenti nelle opere, soprattutto in versi, dell'intera Europa occidentale; si va dalla prova più palmare, cioè la presenza di una notazione musicale che accompagni un componimento, a tracce meno vincolanti come i documenti aneddotici sull'esecuzione di un testo, oppure la presenza all'interno di un'opera di verbi che indicano gli atti di dire e ascoltare riferiti all'opera stessa, o ancora l'accentuata presenza di varianti nella tradizione manoscritta - indizio questo da consi-

Nota: Ringrazio Nello Bertoletti, Mirella Ferrari, Vittorio Formentin, Maria Antonietta Marogna, Paolo Pellegrini, Gabriella Pomaro, Raymund Wilhelm e Massimo Zaggia per i loro suggerimenti.

Indirizzo di corrispondenza: Prof. Dr. Michele Colombo, Romanska och klassiska institutionen, Stockholms Universitet, SE-106 91 Stockholm. E-Mail: michele.colombo@su.se. 
derare con speciale cautela. L'esecuzione orale di un testo poteva assumere diverse forme: il canto, cui si è già accennato, ma anche la recitazione o la lettura ad alta voce; quest'ultima tuttavia, secondo Zumthor, spesso confluiva nella recitazione, perché chi leggeva in pubblico si affidava di frequente alla memoria oltre che agli occhi. In particolare nel caso dei giullari, perciò, è piuttosto elevata la possibilità che l'esecutore, pur avendo davanti a sé un libro dispiegato, lo impiegasse più come accessorio per drammatizzare il discorso che come guida da seguire fedelmente (cf. Zumthor 1990, 82-83, 140). Ne segue un'incertezza di fondo sul testo effettivamente comunicato attraverso l'esecuzione orale, che è certo imparentato con lo scritto giunto fino a noi, ma ad esso non può essere completamente ricondotto, perché «in ogni momento la bocca del lettore, di professione o no, era pronta ad alterarlo, a rimaneggiarlo, a rifarlo» (Zumthor 1999, 134).

Un corollario di grande momento di questa tesi riguarda il rapporto tra cultura popolare e cultura dotta nel Medioevo: opponendosi alle idee di Bachtin (1979) e Gurevič (2007) sull'originalità della cultura popolare e sulla tensione tra essa e la cultura dotta, Zumthor (1990, 39) afferma che, prima del XV secolo, l'aggettivo popolare «non designa ancora quello che si oppone alla «scienza», alla lettrure, ma si riferisce a quello che appartiene a un orizzonte comune a tutti - da cui si distinguono alcune costruzioni astratte proprie di una piccolissima minoranza intellettuale». ${ }^{1}$ È cioè necessario respingere l'associazione tra orale e popolare da un lato, e scritto e colto dall'altro, così come l'erezione di inesistenti paratie stagne tra le diverse classi sociali. Come ha notato, relativamente alla Francia bassomedioevale, Robert Muchembled (1991, 465), molti nobili «vivevano nei paesi e da paesani: la loro era un'esistenza non sempre molto diversa da quella dei contadini abbienti. Infatti prendevano volentieri parte alle feste, indulgevano alle superstizioni e si palesavano in sintonia con la cultura popolare». Lo stesso si può dire con certezza anche del clero e dei religiosi, in particolare degli ordini mendicanti del XIII secolo, ben lontani dal costituire quella casta separata e dedita all'esercizio del potere che a volte è stata ideologicamente tratteggiata.

Con ciò siamo condotti alla considerazione della letteratura religiosa, di cui Zumthor si occupa solo parzialmente, ma che di certo vide una massiccia circolazione orale. Il riferimento non è qui soltanto alla pratica della predicazione, della quale l'oralità costituisce, in certo modo, un carattere connaturale. ${ }^{2}$ Com'è noto, le vite dei santi occupavano una posizione di rilievo nel repertorio non solo dei chierici, ma anche dei giullari, e nella Francia settentrionale del XII secolo è ben documentata la lettura ad alta voce in volgare di testi religiosi (cf. Burgio 2003, 61-62).

1 E cf. anche ibid., 158-159; Barbato (2019, XIII).

2 Se infatti è possibile additare sermoni pensati e stesi solo per l'occhio, essi costituiscono certo un'eccezione che conferma la regola della recitazione a voce (cf. Colombo 2014, 262). 


\section{La Passione del ms. Bergamo, Biblioteca Civica Angelo Mai, MA 460}

Per l'area italoromanza, sulla quale riguardo a questo aspetto gli studi sembrano meno numerosi, è emersa di recente una nuova testimonianza, recata dal manoscritto MA 460 della Biblioteca Civica Angelo Mai di Bergamo. Si tratta di un codicetto membranaceo di soli 23 fogli, più due di guardia, mutilo del foglio originariamente collocato tra il nono e il decimo; le dimensioni, poco più di 20 centimetri in altezza e quindici in larghezza, sono piuttosto ridotte, e nel complesso le caratteristiche indicano un manufatto di uso corrente, senza pretese di lusso. La scrittura - una littera textualis di unica mano ed esecuzione veloce e semplificata, con iniziali rosse e blu, è databile alla seconda metà, probabilmente verso la fine, del XIV secolo, secondo le perizie paleografiche di Mirella Ferrari e Gabriella Pomaro. L'unico testo tramandato dal manoscritto è un racconto della Passione di Cristo che armonizza in un'unica narrazione i quattro vangeli canonici, interpolandoli con inserti esegetici che possono essere ricondotti alla temperie scolastica, dato l'impiego di distinctiones che vi si riscontra, come nel seguente passo, tratto dal f. 20r-v:

Quando elo disse «Sitio», el fo a dire ch'elo aveva sede, e sì disse miser Iesù Cristo per tre rason ch'elo aveva sede. La prima fo natural, che verasiamente elo aveva sede [...]. L'altra rason fo spiritual, a mostrar che tuto çò ch'elo sostigniva, elo lo sostegniva per sede, çò è per desiderio de la salvacion de la humana generacione [...]. La terça rason sì fo açò ch'elo sostegnisse pena in la lenga et in lo gosto sì como in tuti li altri sensi et in tute le altre menbre. ${ }^{3}$

'Quando disse «Sitio», significò che aveva sete, e il signore Gesù Cristo disse che aveva sete per tre ragioni. La prima fu naturale, che aveva effettivamente sete. L'altra ragione fu spirituale, per mostrare che tutto ciò che sopportava, lo sopportava per sete, cioè per desiderio della salvezza del genere umano. La terza ragione fu acciocché penasse nella lingua e nel gusto come in tutti gli altri sensi e in tutte le altre membra'.

Il testo del manoscritto bergamasco - che d'ora in poi chiamerò «Passione Mai» - è da intendere probabilmente come un volgarizzamento dal latino, per la medesima ragione che, in altra sede, ho addotto a proposito di una Passione armonizzata in milanese antico imparentata con quella di cui qui ci si occupa (Colombo 2016, 4-8): dal momento che nel XIV secolo erano disponibili numerose armonie evangeliche in latino (cf. Vaccari 1931, 330-331), è inverosimile,

3 Si sciolgono le abbreviazioni in corsivo, si separano e si uniscono graficamente le parole secondo le norme comunemente in uso per gli antichi testi in volgare, si segnalano tra parentesi uncinate le integrazioni, si distinguono $u$ e $v$, si modificano punteggiatura e maiuscole e si introducono apostrofi e accenti (per la loro qualità ci si riferisce a Gambino 2007; si distinguono $\grave{a}$ 'ha' e $a$ preposizione). 
benché naturalmente possibile, che l'estensore del testo l'abbia non solo volgarizzato, ma anche allestito intrecciando ex novo i passi dei Vangeli. Soprattutto se si pensa al grado di perizia con cui i singoli particolari delle narrazioni evangeliche sono fusi tra loro, come nel caso della predizione del rinnegamento di Pietro:

«Fiioli mei, elo devignirà cusì de vui como fa de le pegore quando lo pastor è morto, le qual tute se desparte. Quando eo serai piiado, vu ve scandaliçerì tuti e tuti scanperì e perderì la mia fe' e lo mio amor, e sì me laxerì solo in man de li pecatori». Quando san Pero aldì questa parola, elo se spaurì tuto e disse: «Signor mio, quando tuti li altri se scandaliçasse en ti, e no me scandaliçerai mai eo», ${ }^{4}$ et ancora disse: "O Iesù Cristo, mo que è questo che tu dis? Che se tuto lo mondo t'abandonasse, eo no t'abandonerai mai, anço sonto aprestado de vegnir cum ti in carcere et in morte». Disse lo Signor: «Pero, Pero, no te mostrar cusì fervente, ch'io te digo in veritade che in questa note, anançi che lo gallo cante doe fiade, tu me negeras tre fiade». Disse santo Pero: «Miser, que è quelo che tu dis, ch'io te negerai? Mo eo me laxerave ananti morire!». E tuti li altri disipoli disse: «Nui no t’abandoneremo mai, miser, anço semo aprestadi de conbatre per ti, s'elo serà bisogno. Echo che nu avemo chialogo dui gladii, cum li qual nu te defenderemo». E lo Signor disse: «Sufficit» (f. 2v).

'«Figli miei, avverrà di voi come delle pecore che, quando il pastore è ucciso, si disperdono tutte. Quando sarò catturato, vi scandalizzerete tutti e scapperete tutti e perderete la mia fede e il mio amore, e mi lascerete solo in mano dei peccatori». Quando san Pietro sentì questo discorso, si turbò tutto e disse: «Signore mio, quandanche tutti gli altri si scandalizzassero di te, io non mi scandalizzerò mai», e inoltre disse: «O Gesù Cristo, che cosa dici? Che se tutti ti abbandonassero, io non ti abbandonerò mai, anzi sono pronto a venire con te in carcere e alla morte». Disse il Signore: «Pietro, Pietro, non ti mostrare così acceso, che ti dico in verità che questa notte, prima che il gallo canti due volte, tu mi rinnegherai tre volte». Disse san Pietro: «Signore che cosa dici, che ti rinnegherò? Ma io mi lascerei piuttosto uccidere!». E tutti gli altri discepoli dissero: «Noi non ti abbandoneremo mai, Signore, anzi siamo pronti a combattere per te, se occorrerà. Ecco che abbiamo qui due spade, con le quali ti difenderemo». E il Signore disse: «Sufficit»’

Il passo segue nella sostanza la narrazione di Matteo (26, 31-35) e Marco $(14,27-31)$, che corrono paralleli, sebbene si possa notare che «vu ve scandaliçerì tuti» è tratto da Marco (14, 27: «omnes scandalizabimini in nocte ista») $)^{5}$ piuttosto che da Matteo (26, 31: «omnes vos scandalum patiemini in me in ista nocte»), e all'inverso «quando tuti li altri se scandaliçasse en ti, e no me scandaliçerai mai eo» risale a Matteo (26, 33: «et si omnes scandalizati fuerint in te, ego numquam scandalizabor») invece che a Marco (14, 29: «et si omnes scandalizati fuerint, sed non ego»). Si attingono però da Giovanni $(16,32)$ la pericope «tuti scanperì [...],

4 Sebbene non si sia condotto uno spoglio sistematico, la forma del pronome di $1^{\text {a }}$ persona $e$, pare assente dal testo; sembra perciò consigliabile vedere qui un caso di paraipotassi.

5 Si cita dalla Vulgata, introducendo la punteggiatura. 
e sì me laxerì solo in man de li pecatori», da Luca la risposta «sonto aprestado de vegnir cum ti in carcere et in morte» $(22,33)$, da Marco (contro Matteo e Luca) il dettaglio del doppio canto del gallo, di nuovo da Luca $(22,38)$ l'affermazione dei discepoli «Echo che nu avemo chialogo dui gladii», legata senza soluzione di continuità al loro accodarsi alla spavalderia di Pietro.

Che il testo sottostante la Passione Mai sia latino e non, per esempio, in francese antico, è suggerito per un verso dall'assenza di armonie evangeliche di area galloromanza (Hoogvliet 2013, 293), per l'altro dagli inserti latini nel dettato, come mostrano per esempio i passi citati sopra («Quando elo disse «Sitio»», «E lo Signor disse: 〈Sufficit»»).

\section{La «Passione Mai» e la lettura ad alta voce}

Una delle caratteristiche che rendono la Passione Mai specialmente degna di attenzione è il lungo prologo che precede la narrazione evangelica vera e propria, dal quale si apprende innanzitutto che il codice era impiegato per la lettura ad alta voce nel venerdì della Settimana Santa:

La passione de miser Iesù Cristo çaschadun fedel cristiano la dé venerar e plançere cum gran devocione e cum gran contricion de core e dé sparçer lagreme devotissime per quelo dolce Cristo, lo qual ancoy sparse lo so sangue glorioso su lo legno de la croxe per nui miseri pecatori, çò fo lo venerdì santo. In quello dì propriamente se leçe e se predica questa gloriosa passione e sì comença questa passione su modo de predication (f. 1r).

'La passione del signore Gesù Cristo ciascun fedele cristiano la deve venerare e piangere con grande devozione e grande contrizione di cuore, e deve spargere lacrime devotissime per quel dolce Cristo che effuse il suo sangue glorioso sul legno della croce per noi miseri peccatori oggi, cioè il venerdì santo. In quel giorno propriamente si legge e si predica questa gloriosa passione, e comincia questa passione al modo di una predica'.

Già queste poche righe iniziali presentano un indizio di oralità eccezionalmente esplicito, certo assai più delle pur convincenti prove addotte da Zumthor (1990, 309-313) per dimostrare la pratica della lettura ad alta voce. Riprendendo i risultati di un'indagine di Verlato (2017, 105 n. 30), infatti, si può notare come di solito la destinazione di un codice volgare alla lettura comunitaria sia solo intuibile «sulla base della qualità dei testi [da esso tramandati], e della loro più o meno evidente funzione divulgativa», piuttosto che apertamente asseribile, come capita invece nel caso della Passione Mai.

Fanno eccezione tuttavia i libri delle confraternite (in specie di battuti), i quali spesso suppongono, oltre al canto comune delle laudi, una voce guida per le preghiere, della quale possono essere specificate anche le parole da proferire 
per introdurre le orazioni, come accade per esempio nel quattrocentesco codice dei battuti bresciani di San Cristoforo:

Preces dicende per ministrum aut subministrum in fine processionis antequam fiat confessio generalis. Carissem frathey me e seror mii, pregé De digant cum e dirò mi devotament. $\mathrm{O}$ Yhesu Christ, el qual nascest de la beatha virgen madona sancta Maria, e che e' veras De e veras hom... (Bonelli/Contini 1935, 119-120).

Anche la Passione Mai propone, in forma più ampia, una sorta di copione, come si può notare proseguendo la lettura del prologo:

Dise e parla quelui che dé predicare de questo dì santissimo e recitare la passion de miser Iesù Cristo: «Signori eo no sai çò che sia ancoy meio fare, o plançere o predicare, ${ }^{6}$ che quando eo inpenso como miser Iesù Cristo, lo nostro Signor, lo nostro salvador, lo nostro pare, lo nostro fradelo, è ancoy morto e crucificado, certo el non è alguna criatura che no debia ancoy plançere, perché è ancoy morto lo fiiol de Dio. ${ }^{7}$ Mo quando eo vego cotanta bona çente esere assenblada per aldire la passione de lo Salvadore, a mi par eser covegnivele cosa no solamente plançere, may eciandio predicare. ${ }^{8}$ Unde nui faremo cusì, che nu plançeremo cum lo core e cum li ogli, se Dio ven darà la gracia, e cum le oregle aldiremo e cum la bocha predicheremo la passione de lo nostro Salvadore» (f. $1 \mathrm{r}-\mathrm{v}){ }^{9}$

'Dice e proclama colui che deve predicare a proposito di questo giorno santissimo e recitare la passione del signore Gesù Cristo: «Signori io non so ciò che oggi sia meglio fare, piangere o predicare, che quando penso a come il signore Gesù Cristo, nostro signore, nostro salvatore, nostro padre, nostro fratello, è oggi morto e crocifisso, certo non c'è nessuna creatura che oggi non debba piangere, perché oggi è morto il figlio di Dio. Ma quando vedo tanta buona gente essere riunita per udire la passione del Salvatore, mi sembra essere buona cosa non solo piangere, ma anche predicare. Dunque faremo così, che piangeremo con il cuore e con gli occhi, se Dio ve ne darà la grazia, e con le orecchie udremo e con la bocca predicheremo la passione del nostro Salvatore»'.

Si tratta, come si vede, di parole letteralmente messe in bocca al lettore perché si rivolga al suo pubblico, suggerendo l'atteggiamento da assumere nei confronti del testo che sta per essere ascoltato. ${ }^{10}$ Prima però di giungere al racconto vero e

6 Con le lettere Signori eo e o predicare ripassate in inchiostro nero da altra mano.

7 Con la $n$ di plançere, la $p$ e l'abbreviazione di per e le lettere $e$ ancoy morto ripassate in inchiostro nero da altra mano.

8 Con cosa no solamente plançere, may (tranne sol-) ripassati in inchiostro nero da altra mano.

9 Con le lettere uen darà la gracia. e ripassate in inchiostro nero da altra mano.

10 Si noti di sfuggita che il riferimento alla predicazione non implica che il lettore fosse necessariamente un chierico. Nelle confraternite infatti anche ai laici era possibile predicare, come mostra l'esempio di Albertano da Brescia (cf. Powell 1992, 90-120). Si tratterebbe in tale evenienza di un caso simile a quello per cui, in alcune compagnie di disciplinati, un confratello era chiamato a tenere un sermone in occasione di ricorrenze particolari, e in specie nella Setti- 
proprio della passione di Cristo, ci si affida alla protezione della Vergine, senza la cui grazia non si potrebbe convenientemente ascoltare la narrazione evangelica, e si propone una preghiera comune:

Unde inprima nu demanderemo la soa gracia e sì la saluderemo cusì digando: «Ave Maria, gracia plena, Dominus tecum, benedicta tu in mulieribus et benedictus fructus ventris tui. Sancta Maria ora pro nobis» (f. 1v).

Un siffatto procedere richiama alla mente di nuovo i libri confraternali con le loro preghiere, specialmente incipitarie, come quelle del laudario dei battuti di Modena (Elsheikh 2001, 9-10), dove si legge ai ff. 14v-15r:

Tut' i sancti e le sancte de Deo [...] sì sianno anchoi a prego cum la madre nostra de vita eterna madonna sancta Maria, [...] açoché quinoga nu possemo desponere gi nostri chori, le mente, le effecto e la voluntae a fare penitencia di nostri peccae [...]. Et açoe che questa nostra madre più voluntera nostra advocata sia, façemoie tuti reverentia digando: Ave Maria gratia plena.

'Tutti i santi e le sante di Dio siano oggi in preghiera con la nostra madre di vita eterna madonna santa Maria, acciocché qui noi possiamo disporre i nostri cuori, le menti, le inclinazioni dell'animo e la volontà a fare penitenza dei nostri peccati. E acciocché questa nostra madre più volentieri sia nostra avvocata, facciamole tutti onore dicendo: Ave Maria gratia plena'.

Proprio nei codici confraternali, non sorprendentemente, si può trovare il racconto della Passione, in verso o in prosa, sia in forma relativamente ridotta, come nel quattrocentesco laudario dei battuti di Novara (Longo 1986, 346-352), sia in forma più estesa, come nel libro dei battuti di Lodi (Agnelli 1902, 57-99; cf. Andreose 2008, 43-45). Non è insomma impossibile che la Passione Mai fosse un testo da impiegare in ambito confraternale, benché tale nesso si riduca semplicemente a una ipotesi che occorre lasciare in sospeso per insufficienza di prove: resta il fatto che essa pare presupporre una esecuzione che si attenga molto da vicino alla lettera del codice. Quel divario che sempre intercorre tra lo scritto giunto fino a noi e la sua trasmissione orale, mai colmabile completamente e con assoluta certezza, sembra perciò nel caso che qui si discute particolarmente sottile.

mana Santa; per esempio, relativamente al XV secolo, si hanno notizie riguardanti i battuti del Duomo di Padova e di S. Domenico a Firenze, i cui statuti prescrivevano che uno dei confratelli tenesse un sermone in una o più delle adunanze previste per la settimana santa (Meersseman 1977, 1274-1275). 


\section{Localizzazione linguistica della «Passione Mai»}

È dunque interessante dedicarsi all'esame della lingua del testo, a partire dal tentativo di localizzarlo dal punto di vista geografico. Per ragioni di spazio, si proporrà qui una descrizione non esaustiva, sia per i fenomeni citati sia per lo spoglio, condotto solo parzialmente. La convergenza di alcuni indizi suggerisce che si abbia a che fare con un testo di area veneta: in tal senso orientano il buono stato di conservazione delle vocali atone finali diverse da $-a$, su cui si tornerà a breve, il dileguo dell'occlusiva dentale in posizione intersonantica, che si riscontra in mare 'madre' 1v, 2r (2 occ.), etc., pare 'padre' 1r, 2r, etc. o Pero 'Pietro' 2r (3 осc.), etc. e l'esito assimilato e poi scempiato del nesso intervocalico ст, riconoscibile in dretamente 'drittamente' $2 \mathrm{r}$, fato 'fatto' $1 \mathrm{r}$ ( 2 occ.), note 'notte' $2 \mathrm{v}$, pato 'patto' 1v, trata 'tratta' 1r, etc. (cf. Arcangeli 1990, 16-17; Marcato 2002, 299; Loporcaro 2009, 102-104).

All'interno del dominio linguistico veneto, diversi fenomeni parlano in favore del veneziano: per il vocalismo tonico, si può notare che il passaggio metafonetico di $e$ a $i$ e di $o$ a $u$ per influsso di una originaria -I finale si restringe a poche forme pronominali. Esemplificando dai due primi fogli del codice, si trovano ili $1 \mathrm{v}, 2 \mathrm{r}$, illi $2 \mathrm{r}$ (2 occ.), quisti $1 \mathrm{v}$ per la vocale palatale e dui $2 \mathrm{v}(2 \mathrm{occ}$.), $n u$ 1r-v, $2 \mathrm{v}(2$ occ.), nui $1 \mathrm{r}$ ( $2 \mathrm{occ}$.), $1 \mathrm{v}$ ( $2 \mathrm{occ}$.), $2 \mathrm{r}-\mathrm{v}$, vu $2 \mathrm{r}$, vui $1 \mathrm{v}$ per la velare, mentre restano preservati amaistramenti $2 \mathrm{r}(3 \mathrm{occ}$.$) , comandamenti (2v), comandamenti (2v), peca-$ tori $1 \mathrm{r}, 2 \mathrm{v}$ ( 2 occ.) e signori 1r, a differenza di quanto ci si aspetterebbe a Belluno, Padova, Treviso o Verona. ${ }^{11}$ Il quadro è però screziato da forme come multi $3 \mathrm{r}$ e signi $5 \mathrm{r}$, a fronte di molto $2 \mathrm{r}$ ( 2 occ.) e segno $5 \mathrm{r}$, e soprattutto dal diffuso esito metafonetico in - $i$ della desinenza verbale di $5^{a}$ persona -ETIS, assai raro a Venezia, riconoscibile nel presente indicativo savi 'sapete' $2 \mathrm{r}$ e nei futuri contenuti nella frase con cui Gesù predice agli apostoli la loro fuga: «Quando eo serai piiado, vu ve scandaliçerì tuti e tuti scanperì e perderì la mia fe' e lo mio amor, e sì me laxerì solo in man de li pecatori» (2v) ('Quando sarò pigliato, voi vi scandalizzerete tutti e scapperete e perderete la fede e l'amore in me, e mi lascerete solo in mano dei peccatori'). ${ }^{12}$

11 Cf. Salvioni (1894, 308-311); Stussi (1965, xxxvII-XxxIx); Ineichen (1962-1966, vol. 2, 360-361); Brugnolo (1977, 143-145); Pellegrini (1977, 316); Donadello (1994, 42); Stussi (1995, 127, 130-132); Tomasoni (1973, 172-173); Tomasin (2004a, 100-102); Bertoletti (2005, 42-53; 2006, 13, 25). Ma si noti che nel bellunese e nel trevisano antichi la metafonesi è assente nei pronomi personali di $4^{\mathrm{a}}$ e $5^{\mathrm{a}}$ persona (cf. Formentin 2005, 311).

12 Per la $5^{\text {a }}$ persona, nei testi pratici veneziani pubblicati da Stussi (1965, XxxvIII) si trova solo un «isolato seri [...] (di solito seré)». 
Significativa è la condizione di caduta delle atone finali, tra le quali la palatale - $e$ può dileguarsi dopo $-n$, $-l$ e $-r$, la velare - $o$ dopo $-n$ in parole piane e dopo $-l$ nel suffisso -ol. Nei primi due fogli del codice, la - $e$ cade dunque dopo $-n$ in contricion 1r, passion 1v (3 occ.), passion 1r (2 occ.), 1v, 2r, predication 1r; dopo $-r$ in amor 2r (2 occ.), miser 'messere' 1r (4 occ.), 1v (3 occ.), 2r (3 occ.), miser 1r (2 occ.), $1 \mathrm{v}, 2 \mathrm{r}$, salvador 1r, signor 1r, 1v (2 occ.), 2r (3 occ.), vener 'venerdi' 1v, nel presente indicativo par 1r e negli infiniti aver 2r, eser 1r, sparçer 1r, venerar 1r; cade infine dopo -l negli aggettivi e pronomi fedel 1r, qual 1r (3 occ.), 1v (3 occ.), 2r e tal 1v. Si noti che non si ha caduta di -e né quando la sonorante che precede faceva parte di un gruppo consonantico, come nei già citati mare da MATRE(M) e pare da PATRE(M), né quando si tratta di morfema del plurale, come in maynere $1 \mathrm{v}$. Per quanto riguarda la -o, essa vanisce in bon 2r (2 occ.), çaschadun 1r e nel sostantivo fiiol 1r. Gli esempi menzionati si affiancano ad altri in cui invece le finali sono conservate, ma quel che importa è che non si trovano casi di caduta dopo consonanti diverse dalle sonoranti $n$, $l$ e $r$, che condurrebbero lontano da Venezia, verso il Veneto settentrionale; d'altro canto, la caduta delle finali dopo le alveolari $-l \mathrm{e}-r$ esclude Padova, dove il fenomeno si manifesta solo in presenza di un condizionamento fonosintattico, come in Toscana, e la mancanza di neutralizzazione in -o della -e finale non permette di orientarsi verso Verona. ${ }^{13}$

Caratteristici di Venezia sono inoltre l'esito in -er(o) del suffisso -ARIUM, che si apprezza per esempio in dineri 'denari' $1 \mathrm{v}$, 9v; l'esito in labiodentale sonora $v$ - del germanico $\mathrm{W}$ - a inizio parola, per cui si possono produrre varire 'guarire' 3v (< fr.a. * guarir < franc. * warjan) o vardare $7 \mathrm{r}$, vardar $8 \mathrm{r}$ 'guardare' $\left(<{ }^{\star}\right.$ wardōn $)$, varda 12r, etc., accanto però a un maggior numero di forme con esito labiovelare: guarido $6 \mathrm{v}$ e guarda $6 \mathrm{r}$, guardado $1 \mathrm{v}$, guarderà $4 \mathrm{v}$, etc.; e l'estensione del morfema verbale di $4^{\mathrm{a}}$ persona -emo anche al presente indicativo di essere e dei verbi di prima coniugazione, come in semo $2 \mathrm{v}$ e in demandemo $6 \mathrm{r}$ e aidemo 6r; propria del veneziano più antico (duecentesco e di primo Trecento) è poi l'assenza dell'articolo definito el e l'impiego esclusivo di lo (Stussi 1965, XXXIX, XLIV-XLV, LX; Stussi 1995, 128-129). ${ }^{14} \mathrm{Il}$ dato più rilevante è la folta attestazione di $2^{\mathrm{e}}$ persone verbali desinenti in -s, fenomeno che costituisce, per citare Alfredo Stussi $(2005,72)$, «uno dei tratti più individualizzanti del veneziano»: lo si incontra nell'indicativo

13 Cf. Salvioni (1894, 313-314); Stussi (1965, xxxIII-Xxxv); Ineichen (1962-1966, vol. 2, 364-365); Brugnolo (1977, 169-172); Pellegrini (1977, 358); Donadello (1994, 42); Stussi (1995, 128, 131-132); Tomasoni (1973, 176); Tomasin (2004a, 124-127; 2004b, 168); Bertoletti (2005, 116-137; 2006, 8, 14, 25).

14 In Stussi (1965, LX), gli esiti di w- sono ritenuti privi di valore distintivo nell'area veneta. Diverso il giudizio in Stussi $(1995,129 ; 2005,71)$, dove si considera il passaggio di w- in $v$ - come caratteristico del veneziano. 
presente dis 'dici' (2 occ.) e nel futuro negeras 'negherai' all'interno del dialogo già citato in cui Gesù predice a Pietro il suo rinnegamento:

«O Iesù Cristo, mo que è questo che tu dis? Che se tuto lo mondo t’abandonasse, eo no t'abandonerai mai, anço sonto aprestado de vegnir cum ti in carcere et in morte». Disse lo Signor: «Pero, Pero, no te mostrar cusì fervente, ch'io te digo in veritade che in questa note, anançi che lo gallo cante doe fiade, tu me negeras tre fiade». Disse santo Pero: «Miser que è quelo che tu dis, ch'io te negerai? Mo eo me laxerave ananti morire!» (f. 2v).

Al passo citato si può aggiungere che, nei primi cinque fogli del codice, si raccolgono abis 'tu abbia' 5v, dormis 'dormi' 4r, es 'sei' 5r (3 occ.), fas 'fai' 5r, oferis 'offri' 5r, podis 'puoi' 4r e vos 'vuoi' 3v.

Per quanto riguarda la collocazione cronologica del testo, sembra significa-

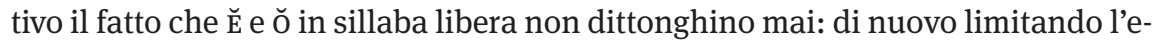
semplificazione ai primi due fogli del manoscritto, per la serie palatale si hanno dè 'diede' 2r (2 occ.) e dèlli 'gli diede’ 2r, dredo 'dietro' 2r, eri 'ieri' 1v, mei 'miei' 2r, pertene 'pertiene' 1v (2 occ.), pei 'piedi' 1v, 2r e Pero 'Pietro' 2r (3 occ.), oltre che bene $2 \mathrm{r}$, da citare a parte vista la sua refrattarietà al dittongo. Per la serie velare si trovano bon 'buono' 2r (2 occ.), con cui vanno bona 1r e boni 2r, core 'cuore' 1r (2 occ.), 1v, fiiol 1r e fiiolo 1v 'figliolo', con il plurale fiioli 2r, mo 1r, 1v (2 occ.) e modo 1r, 2r da MŏDo. ${ }^{15}$ Il fatto si spiegherebbe se la Passione Mai - tramandata, lo si è detto, da un codice del tardo Trecento - fosse copia di un testo precedente di qualche decennio la realizzazione del manoscritto: i dittonghi in sillaba libera infatti, inizialmente assenti in veneziano, si diffondono per gradi, risultando stabilmente acclimati verso la metà del XIV secolo. ${ }^{16} \mathrm{~A}$ una datazione del testo alla prima metà del Trecento spingerebbe anche il già citato impiego esclusivo dell'articolo definito lo, visto che la coesistenza con el si afferma solo verso la metà del secolo. ${ }^{17}$

15 All'elenco si aggiunge forse iveloga 'll' 2r, se l'etimologia da IBI + una forma ricostruita *īLLŏco con o breve è corretta (cf. FEW, vol. 4, 559-560; Rohlfs 1969, §909; VSES, vol. 1, 354-355).

16 Cf. Stussi (1965, XxxIX-XLIII); Sattin (1986, 62-65); Stussi (1995, 127-128); Tomasin (2001, 175; 2010, 58-59; 2012, 31; 2013, 7); Ferguson (2015, 29); Formentin (2018, 436, con rinvio all'indice dei fenomeni e dei temi; si noti che all'assenza di dittongo di $\breve{~}$ e ŏ in sillaba libera in tutti i testi duecenteschi commentati risponde la «dittongazione ormai generale» nella lettera di Cataruza da Pesaro della seconda metà del Trecento: ibid., 341).

17 Cf. Stussi (1965, Xliv); Sattin (1986, 101-103); Stussi (1995, 129); Tomasin (2010, 60; 2012, 31; 2013, 10); Formentin $(2018,55,80,200,247,252,264)$. Le preposizioni articolate però non si presentano nella forma forte in -o, come si nota in testi veneziani duecenteschi (ibid., 62, 87, 118, 228, 252, 282, 317): la situazione trova corrispondenza nella prima redazione della copia della lettera di Ghazan, ilkhan di Persia, al Doge di Venezia, del 30 dicembre 1300, vergata in un volgare veneziano in cui si rileva «la pervasiva presenza dell'elemento francese» (ibid., 308). Il parallelo 
Stabilita la sostanziale pertinenza veneziana della Passione Mai, andrà aggiunto che alcuni suoi caratteri linguistici non si addicono propriamente a tale collocazione. È il caso di un participio tronco come conceù 'concepito' 2r, di contro alla conservazione della vocale finale in iato nel veneziano, e soprattutto della già citata presenza dell'esito metafonetico in -ì della desinenza verbale -ETIS, come in savì, scandaliçeri, etc., tratti che tradiscono un influsso non rialtino, forse padovano (cf. Ineichen 1962-1966, vol. 2, 361 n. 1, 401; Stussi 1995, 130-131; Tomasin 2004, 185). ${ }^{18}$

Anche conteggiando tali eccezioni, e qualcun'altra di minor momento che qui per la tirannia dello spazio si tralascia di discutere, è comunque ragionevole ritenere che la Passione Mai sia un testo in veneziano antico idiomaticamente piuttosto ben caratterizzato. Un testo, si diceva, concepito per essere letto ad alta voce (almeno) una volta all'anno, per il quale - mantenendo la necessaria prudenza - si può però supporre una buona corrispondenza di massima tra testimonianza scritta ed esecuzione orale.

vale solo per affermare che, probabilmente, la compresenza della forma forte dell'articolo definito e di preposizioni articolate apocopate è un carattere non rialtino, che va ad aggiungersi a quelli citati qui oltre.

18 A Padova potrebbero pertenere anche sonto $2 \mathrm{v}$ (e sont'eo vegnudo nello stesso foglio), $5 \mathrm{v}$ (due volte), etc., $1^{\text {a }}$ persona del presente indicativo del verbo essere, attestata ai vv. 2, 3 e 9 del sonetto Paduanus della tenzone tridialettale di Nicolò de' Rossi (Brugnolo 1996, 18), nella Bibbia istoriata (in frase interrogativa con vocale elisa: sont'e'; cf. Donadello 2006, 114) e nel Serapiom (Ineichen 1962-1966, vol. 2, 399), e l'avverbio anço 'anzi' 2v (3 occ.), etc., che ricorre nel Frammento Papafava (Contini 1960, vol. 1, 807) e nel Serapiom (Ineichen 1962-1966, vol. 1, 147); devanzo, enanço ed enanzo sono inoltre attestati nei testi pratici padovani trecenteschi (Tomasin 2004a, 127), enanço, inanço o innanço nella Bibbia istoriata (Folena/Mellini 1962, 127), denanço e innanço nel Serapiom (Ineichen 1962-1966, vol. 1, 402-403). Ma forse, una volta ancora, è più prudente fermarsi a rilevare che tali forme non sono rialtine, visto che sonto e anço si trovano pure altrove sia in Veneto - per es. a Verona - sia oltre i confini del Veneto. Anche nella Legenda de santo Stadi del veneziano Franceschino Grioni, scritta tra la fine del XIII e il principio del XIV secolo, ma tramandata da un codice del secondo quarto del Quattrocento, si trovano $5^{\mathrm{e}}$ persone in -ì e participi in -ù (Monteverdi 1930, 31-32; Badas 2009, XLII, LXXIV, CI; sull'edizione Badas cf. i rilievi di Lorenzi 2011, che comunque non toccano i punti qui rilevanti). Forme metafonetiche come misi 'mesi' e infirmi e participi in -ù si riconoscono anche nella seconda delle mani che verga il trecentesco Capitolare dei Camerlenghi di Comun e che Tomasin (1999, 29-30) giudica perciò non rialtina. 


\section{Tratti fonetici cólti nella «Passione Mai»}

In questa prospettiva, mi pare interessante badare non solo ai caratteri che individuano il volgare adoperato e lo legano a una lingua d'uso quotidiano, ma anche a quei tratti fonetici cólti che spesso sono intesi semplicisticamente come un rumore che disturba la ricezione del vernacolo puro. Nel caso della Passione Mai, infatti, siamo senz'altro nell'àmbito di quella che si potrebbe chiamare, rifacendosi al modello di Peter Koch e Wulf Oesterreicher, un’oralità elaborata («elaborierte Mündlichkeit»), nella quale all'impiego della voce non corrisponde un'immediatezza comunicativa, ma piuttosto una distanza che si mostra in vari aspetti, tra cui il fatto che la comunicazione sia pubblica, che il suo tema sia fisso, che si tratti - a quanto pare - di un monologo e soprattutto che il testo sia frutto di una riflessione precedente che possiamo supporre approfondita. ${ }^{19}$ Coerentemente con tali caratteristiche, la lingua della Passione Mai è aperta alla presenza di forme latineggianti che si staccano dalla pronuncia più consona all'area idiomatica veneta. Nel trattare di questo versante della lingua del testo, è utile distinguere con chiarezza ciò che potremmo chiamare «latineggiamento fonetico», del quale qui ci si occupa, dal latinismo, cioè dalla parola di tradizione dotta, entrata nell'uso come prestito.

Il latineggiamento fonetico può verificarsi infatti non solo in parole di tradizione dotta, come clausura, ma anche in parole di tradizione popolare, come fide per fede (esemplifico qui, per semplicità, attingendo e riferendomi all'italiano piuttosto che al veneziano). Si noti inoltre che non necessariamente la presenza di una parola di tradizione dotta comporta caratteristiche fonetiche latineggianti: è il caso per esempio dei superlativi in -issimo, come grandissimo, i quali non hanno nulla che li identifichi come latinismi all'orecchio di chi non sia un linguista (cf. Durante 1981, 95-96; Reinheimer-Rîpeanu 2004, §3.2.2). Ne segue dunque che si possono avere sia parole di tradizione dotta che hanno un'unica forma integralmente latina, come il già citato clausura, sia parole di tradizione dotta che, al pari delle corrispondenti parole di tradizione popolare, possono essere più e meno latineggiate, come mostra la coppia iubilo/giubilo.

Quello che qui interessa sono dunque le parole latineggianti di tradizione sia dotta sia popolare che, all'orecchio di chi ascoltò la Passione Mai, dovevano suonare estranee alle sue abitudini fonetiche. Nel novero rientrano innanzitutto forme che conservano il timbro di I I e Ŭ toniche e atone in parole che in veneziano antico presentavano e e $o$. Per la serie palatale si trovano confirma 2r, e, fuor d'ac-

19 Cf. Koch/Oesterreicher (1985, 23, 30). Per una applicazione specifica del modello comunicativo ai testi antichi cf. Koch (1993), in part. il §5, La scripturalité à destin vocal. 
cento, infirmitade pl. 16v, infirmitade pl. 16v, humilitade $5 \mathrm{r}, 5 \mathrm{v}$ (2 occ.), 7v, e virtude $16 \mathrm{v}$ (2 occ.), etc. attestato allato a vertude 16v. Per la serie velare è da citare, con $u$ in posizione tonica, il connettivo unde (esclusivo all'interno della Passione Mai), mentre in atonia si hanno la preposizione cum, forma anch'essa esclusiva, dulcissimo 10v (allato a dolcissimo 15r), voluntade $8 \mathrm{v}$, il verbo crucificado $1 \mathrm{r}, 11 \mathrm{v}$, etc., in opposizione a croxe, e titulo $16 \mathrm{v}$ (2 occ.), 17r (3 occ.). Da citare inoltre i casi di mantenimento di [j] iniziale come iustamente $17 \mathrm{r}$, iusto $9 \mathrm{v}$, iusticia $6 \mathrm{r}$ (2 occ.), a fronte dei possibili çustamente o çustamentre, çustiçia e çusto.

Quanti dei tratti fonetici latineggianti tramandati dalla grafia saranno stati davvero pronunciati nella lettura ad alta voce? Il dubbio è più che lecito, soprattutto se si pensa al fatto che, con ogni probabilità, una grafia come clamà $5 r$ (2 occ.) poteva sottintendere una pronuncia con affricata palatale sorda ([ta'ma]). Inoltre va tenuto presente che, nel complesso, il tasso di latineggiamento della Passione Mai non è per nulla ingente, il che conferma, per altro verso, la sua buona caratterizzazione vernacolare. D’altra parte, se è piuttosto rischioso azzardare un giudizio sul modo di pronuncia di una singola parola di un testo medioevale, sarebbe antieconomico ritenere che tutti i latineggiamenti fonetici che la grafia tramanda venissero sistematicamente disattesi nella lettura.

Qual è lo scopo di una simile osservazione? Naturalmente non si scopre nulla di nuovo rimarcando l'influsso del latino sulla facies linguistica di un testo volgare medioevale. E tuttavia non è inutile ribadire come esso si iscriva nella più generale opportunità di non separare artificiosamente cultura popolare e cultura dotta, di cui si diceva in principio seguendo Zumthor. È una opportunità che mi pare valida sia sul piano del contenuto sia su quello della forma, perché anche nell'esecuzione orale di un testo non è possibile negare, e anzi è necessario accordare, diritto di cittadinanza agli elementi dotti così come a quelli popolari.

\section{Riferimenti bibliografici}

Agnelli, Giovanni, Il libro dei battuti di San Defendente di Lodi. Saggio di dialetto lodigiano del secolo decimo quarto, Archivio Storico per la Città e Comuni del Circondario di Lodi 21 (1902), I-xv, 1-108.

Andreose, Alvise, Censimento dei testimoni della «Lamentatio Beate Virginis» di Enselmino da Montebelluna. II, Quaderni Veneti 47-48 (2008), 9-98.

Arcangeli, Massimo, Per una dislocazione tra l'antico veneto e l'antico lombardo (con uno sguardo alle aree contermini) di alcuni fenomeni fonomorfologici, L'Italia Dialettale 53 (1990), 1-42.

Bachtin, Michail, L'opera di Rabelais e la cultura popolare. Riso, carnevale e festa nella tradizione medievale e rinascimentale, Torino, Einaudi, 1979 [Tvorčestvo Fransua Rable $i$ narodnaja kul'tura srednevekov'ja Renessansa, Moskva, Izdatel'stvo «Chudožestvennaja literatura», 1965]. 
Badas, Mauro (ed.), Franceschino Grioni, La legenda de santo Stadi, Roma/Padova, Antenore, 2009.

Barbato, Marcello (ed.), Incantamenta latina et romanica. Scongiuri e formule magiche dei secoli $V-X V$, Roma, Salerno Editrice, 2019.

Bertoletti, Nello, Testi veronesi dell'età scaligera, Padova, Esedra, 2005.

Bertoletti, Nello, Testi in volgare bellunese del Trecento e dell'inizio del Quattrocento, Lingua e Stile 41 (2006), 3-26.

Bonelli, Giuseppe/Contini, Gianfranco, Antichi testi bresciani, L'Italia Dialettale 11 (1935), 115-151.

Brugnolo, Furio, Il canzoniere di Nicolò de’ Rossi, vol. 2: Lingua, tecnica, cultura poetica, Padova, Antenore, 1977.

Brugnolo, Furio, Ritornando sulla canzone di Auliver e su altre liriche di età caminese.

Precisazioni e proposte, Quaderni Veneti 24 (1996), 9-25.

Burgio, Eugenio, Il pubblico della letteratura religiosa nella Francia settentrionale, in: Boitani, Piero/Mancini, Marco/Vàrvaro, Alberto (edd.), Lo spazio letterario del Medioevo, 2: Il Medioevo volgare, vol. 3: La ricezione del testo, Roma, Salerno Editrice, 2003, 53-78.

Colombo, Michele, Predicazione e oratoria politica, in: Antonelli, Giuseppe/Motolese, Matteo/ Tomasin, Lorenzo (edd.), Storia dell'italiano scritto, vol. 3: Italiano dell'uso, Roma, Carocci, 2014, 261-292.

Colombo, Michele, Passione Trivulziana. Armonia evangelica volgarizzata in milanese antico. Edizione critica e commentata, analisi linguistica e glossario, Berlin/Boston, De Gruyter, 2016.

Contini, Gianfranco (ed.), Poeti del Duecento, 2 vol., Milano/Napoli, Ricciardi, 1960.

Donadello, Aulo (ed.), Il libro di messer Tristano ("Tristano Veneto»), Venezia, Marsilio, 1994. Donadello, Aulo, Nuove note linguistiche sulla «Bibbia Istoriata Padovana», in: Brugnolo, Furio/Verlato, Zeno (edd.), La cultura volgare padovana nell'età del Petrarca. Atti del convegno (Monselice/Padova, 7-8 maggio 2004), Padova, Il Poligrafo, 2006, 103-171.

Durante, Marcello, Dal latino all'italiano moderno. Saggio di storia linguistica e culturale, Bologna, Zanichelli, 1981.

Elsheikh, Mahmoud Salem (ed.), Il laudario dei Battuti di Modena. Testo, nota linguistica e glossario, Bologna, Commissione per i Testi di Lingua, 2001.

Ferguson, Ronnie, Le iscrizioni in antico volgare delle confraternite laiche veneziane, Venezia, Marcianum Press, 2015.

FEW = von Wartburg, Walther, Französisches etymologisches Wörterbuch, 25 vol., Tübingen/ Basel, Mohr/Helbing \& Lichtenhahn/Zbinden, 1948-2002.

Folena, Gianfranco/Mellini, Gian Luigi (edd.), Bibbia istoriata padovana della fine del Trecento. Pentateuco - Giosuè - Ruth, Venezia, Neri Pozza, 1962.

Formentin, Vittorio, Sul frammento zurighese di Giacomino Pugliese, Lingua e Stile 40 (2005), 297-316.

Formentin, Vittorio, Prime manifestazioni del volgare a Venezia. Dieci avventure d'archivio, Roma, Edizioni di Storia e Letteratura, 2018.

Gambino, Francesca (ed.), I Vangeli in antico veneziano. Ms. Marciano it. I 3 (4889), Roma/ Padova, Antenore, 2007.

Gurevič, Aron Jakovlevič, Contadini e santi. Problemi della cultura popolare nel Medioevo, Torino, Einaudi, 1986 [Problemy srednevekovoj narodnoj kul'tury, Moskva, Izdatel'stvo «lskusstvo», 1981]. 
Hoogvliet, Margriet, The Medieval Vernacular Bible in French as a Flexible Text: Selective and Discontinuous Reading Practices, in: Poleg, Eyal/Light, Laura (edd.), Form and Function in the Late Medieval Bible, Leiden/Boston, Brill, 2013, 283-331.

Ineichen, Gustav, El libro agregà de Serapiom. Volgarizzamento di frater Jacobus Philippus de Padua, 2 vol., Venezia/Roma, Istituto per la Collaborazione Culturale, 1962-1966.

Koch, Peter, Pour une typologie conceptionnelle et médiale des plus anciens documents/ monuments des langues romanes, in: Selig, Maria/Frank, Barbara/Hartmann, Jörg (edd.), Le passage à l'écrit des langues romanes, Tübingen, Gunter Narr, 1993, 39-81.

Koch, Peter/Oesterreicher, Wulf, Sprache der Nähe - Sprache der Distanz. Mündlichkeit und Schriftlichkeit im Spannungsfeld von Sprachtheorie und Sprachgeschichte, Romanistisches Jahrbuch 36 (1985), 15-43.

Longo, Pier Giorgio, Letteratura e pietà a Novara tra XV e XVI secolo, Novara, Associazione di Storia della Chiesa Novarese/Fondazione Achille Marazza, 1986.

Loporcaro, Michele, Profilo linguistico dei dialetti italiani, Roma/Bari, Laterza, 2009.

Lorenzi, Cristiano, [Recensione a Franceschino Grioni, La legenda de santo Stadi, a c. di Mauro Badas, Roma/Padova, Antenore, 2009], Lingua e Stile 46:2 (2011), 301-312.

Marcato, Carla, Il Veneto, in: Cortelazzo, Manlio, et al., I dialetti italiani. Storia, struttura, uso, Torino, UtET, 2002, 296-328.

Meersseman, Gilles-Gérard, I predicatori laici nelle confraternite medievali, in: id., Ordo Fraternitatis. Confraternite e pietà dei laici nel Medioevo, vol. 3, Roma, Herder, 1977, 1273-1289.

Monteverdi, Angelo, La legenda de santo Stady di Franceschino Grioni, Studj Romanzi 20 (1930), 1-199.

Muchembled, Robert, Cultura popolare e cultura delle élites nella Francia moderna (XV-XVIII secolo), Bologna, il Mulino, 1991 [Culture populaire et culture des élites dans la France moderne (XVe-XVIII siècles), Paris, Flammarion, 1978].

Pellegrini, Giovan Battista, Studi di dialettologia e filologia veneta, Pisa, Pacini, 1977.

Powell, James M., Albertanus of Brescia. The Pursuit of Happiness in the Early Thirteenth Century, Philadelphia, University of Pennsylvania Press, 1992.

Reinheimer-Rîpeanu, Sanda, Les emprunts latins dans les langues romanes, Bucureşti, Editura Universităţii din Bucureşti, 2004.

Rohlfs, Gerhard, Grammatica storica della lingua italiana e dei suoi dialetti, 3 vol., Torino, Einaudi, 1966-1969.

Salvioni, Carlo, Annotazioni linguistiche, in: Cian, Vittorio/Salvioni, Carlo (edd.), Le rime di Bartolomeo Cavassico notaio bellunese della prima metà del sec. XVI, vol. 2, Bologna, Romagnoli Dall'Acqua, 1894, 307-437.

Sattin, Antonella, Ricerche sul veneziano del sec. XV (con edizione di testi), L'Italia Dialettale 49 (1986), 1-172.

Stussi, Alfredo, Testi veneziani del Duecento e dei primi del Trecento, Pisa, Nistri-Lischi, 1965.

Stussi, Alfredo, Venezien/Veneto, in: Holtus, Günter/Metzeltin, Michael/Schmitt, Christian (edd.), Lexikon der Romanistischen Linguistik, vol. II/2, Tübingen, Niemeyer, 1995, 124-134.

Stussi, Alfredo, Medioevo volgare veneziano, in: id., Storia linguistica e storia letteraria, Bologna, il Mulino, 2005, 23-80.

Tomasin, Lorenzo, Il Capitolare dei Camerlenghi di Comun (Venezia, circa il 1330), L'Italia Dialettale 60 (1997-1999), 25-103. 
Tomasin, Lorenzo, La lapide veneziana di S. Gottardo (1384) a Piazzola sul Brenta, L'Italia Dialettale 62 (2001), 173-177.

Tomasin, Lorenzo, Testi padovani del Trecento, Padova, Esedra, 2004 (= 2004a).

Tomasin, Lorenzo, Calendario trecentesco delle feste per la scuola di S. Martino di Belluno, in: Zaccarello, Michelangelo/Tomasin, Lorenzo (edd.), Storia della lingua e filologia. Per Alfredo Stussi nel suo sessantacinquesimo compleanno, Firenze, Edizioni del Galluzzo per la Fondazione Ezio Franceschini, 2004, 159-176 (= 2004b).

Tomasin, Lorenzo, Storia linguistica di Venezia, Roma, Carocci, 2010.

Tomasin, Lorenzo, Epigrafi trecentesche in volgare nei dintorni di Venezia, Lingua e Stile 47 (2012), 23-44.

Tomasin, Lorenzo, Quindici testi veneziani 1300-1310, Lingua e Stile 48 (2013), 3-48.

Tomasoni, Piera, Per una storia dell'antico trevisano, Studi di Grammatica Italiana 3 (1973), 155-206.

Vaccari, Alberto, Propaggini del Diatessaron in Occidente, Biblica 12 (1931), 326-354.

Verlato, Zeno, Vicende di uomini e di libri. Due note tipologiche per la storia del libro agiografico volgare, in: Albonico, Simone/Bock, Nicolas (edd.), Santi, santità e agiografie nell'Italia settentrionale. Percorsi letterari e storico-artistici tra Medioevo e età moderna, Pisa, ETs, 2017, 93-122.

VSES = Vàrvaro, Alberto, Vocabolario Storico-Etimologico del Siciliano, 2 vol., Strasbourg, Éditions de Linguistique et de Philologie/Centro di Studi Filologici e Linguistici Siciliani, 2014.

Vulgata = Biblia sacra iuxta vulgatam versionem, edd. Weber, Robert/Gryson, Roger, Stuttgart, Deutsche Bibelgesellschaft, ${ }^{5} 2007$.

Zumthor, Paul, La lettera e la voce. Sulla «letteratura» medievale, Bologna, il Mulino, 1990 [La lettre et la voix: de la «littérature» médiévale, Paris, Seuil, 1987].

Zumthor, Paul, Una cultura della voce, in: Boitani, Piero/Mancini, Mario/Vàrvaro, Alberto (edd.), Lo spazio letterario nel Medioevo, 2: Il Medioevo volgare, vol. 1/1: La produzione del testo, Roma, Salerno Editrice, 1999, 117-146. 


\section{Indici}

AvVERTENZA GENERALE. Sono esclusi dall'indicizzazione sia i nomi in lingua latina e quelli letterari o di fantasia (e.g. Achille), sia i rinvii bibliografici citati nel corpo del testo, nelle note a piè pagina e nei Riferimenti in calce a ciascun contributo. Le sigle con cui alcuni manoscritti possono essere nominati si riferiscono ai soli contributi compresi nel presente volume; anche alcune segnature di codici si trovano elencate qui di séguito in una forma normalizzata che può risultare leggermente diversa da quella adottata in alcuni dei saggi componenti la miscellanea (in particolare, s'è scelto d'inserire un punto per separare cifre da cifre e uno spazio tra lettere oppure fra lettere e cifre). 



\section{Indice dei nomi}

Acciaiuoli Niccolò IX, 197-199

Adam von Ammergau $92 \mathrm{n}$.

Adriano Publio Elio Traiano 292

Agostino Aurelio, santo 15, 237

Aimone di Faversham 8

Albertano da Brescia 160 n., 320 e n.

Alberti Leon Battista 153

Alessandro III, re di Macedonia, detto Magno 250

Alessio, santo 20-21

Alfonso V d'Aragona, re di Sicilia, re di Napoli 93

Alfonso X il Saggio, re di Castiglia e León 26, 41, 50 n., $220 \mathrm{n}$.

Alighieri Dante 9-10, 200, 244, 273, 275

Alluccio da Pescia, santo 6

Anastasio I, imperatore d'Oriente 283

Andreoni Armando 353

Anonimo romano 211, 217, 225, 226 e n.

Antonio abate, santo 15

Antonio Marco, il triumviro 289

Apollonio di Tiro 353

Aristotele 274

Armannino da Bologna 275, 284, 294

Arnaldo da Villanova 50

Assmann Aleida $214 \mathrm{n}$.

Atto, santo 325

Augusto Gaio Giulio Cesare Ottaviano, imperatore 247, 274-275, 277, 289-292

Azzetta Luca 324

Bachtin Michael 215

Baglioni, Giovanni (Giannino) $287 \mathrm{n}$.

Barbato da Sulmona 206

Barbato Marcello, $215 \mathrm{n}$.

Barbieri Luca X, 337

Barbieri Edoardo 188 n., 191 n.

Bargellini Angela 358

Baroni Pellegrino 294

Bartolo da Sassoferrato 64

Bartolomeo Anglico $22 \mathrm{n}$.

Bartolomeo da San Concordio 90, 238, 255, 268

Bartolomeo di Neocastro 176
Bartromei Guillielmo 327

Bartromei Michele 327

Basilio di Cesarea, detto il Grande, santo 12

Bassetti Massimiliano $319 \mathrm{n}$.

Beccaria, Tesauro 173

Beda, detto il Venerabile, santo 221

Belcalzer Vivaldo $22 \mathrm{n}$.

Belcari Feo 88

Bellebuoni Giovanni 320, 322, 323 e n., 324-327, 346-347

Bellebuoni Mazzeo XI, 319-322, 323 e n., 324, 325 e n., 326 e n., 327, 328 e n., 329, 331, 332 n., 338 n., 339 e n., 340, 344, 345 e n., 346-347, 348 e n., 349, 353-355, 357, 358 e n., 359 e n., 360 e n.

Benedetto da Norcia, santo 12, 48

Benincà Paola 153

Benoît de Sainte-Maure 300, 316, 329 n., 330 n., 332 n., 333, 334 n., 335 n., 336-337

Benzo d'Alessandria $330 \mathrm{n}$.

Berger Samuel 86, 96

Bernardo di Chiaravalle, santo 52

Bernardo di Chiaravalle, santo (pseudo Bernardo) 199

Berti Sara 200

Bertoletti Nello $155 \mathrm{n}$.

Bertucci Fredi 327

Billanovich Giuseppe 202, 358

Binduccio dello Scelto 211, 217, 224-225, 315 n., 333 e n., 338

Blasio Maria Grazia $273 \mathrm{n}$.

Boccaccio Giovanni IX, XI, 9-10, 199, 202 n., 299-302, 315, 316 e n., 317, 339 n.

Boezio Anicio Manlio Torquato Severino 89

Bonaventura da Bagnoregio 11-12

Bonifacio VIII, papa (Benedetto Caetani) 278, 280-281

Bonifacio, vescovo di Ferentino, santo 73

Bonsignori Giovanni 289 e n., 291

Bonvesin da la Riva VIII, 19, 21-22, 23 e n., 24, 25 e n., 26, 27 n., 28, 31 e n., 32-33, 35-36, 37 e n., 63, 76

Borghini Vincenzio 289, 313 
Brambilla Simona IX

Brucioli Antonio 92

Bruner James Dowden 320 e n., 321

Bruni Leonardo, detto Leonardo Aretino 199-200, 274 n., 275, 278 n., 346 n.

Bruto Marco Giunio 289

Burgassi Cosimo $273 \mathrm{n}$.

Caligola 277

Campi Giuseppe 345 n.

Campulu Giovanni 49

Canali Luca $330 \mathrm{n}$.

Canneti Caterina $273 \mathrm{n}$.

Canzio Nicoletta $330 \mathrm{n}$.

Cappi Davide 227 n., 333 n.

Carlesso Giuliana 339 e n., 344 e n.

Carlo I d'Angiò, re di Sicilia IX, 174-175, 180, 182, 186 n., 188-189, 190 e n., 191, 193 e $n$.

Carlo IV di Lussemburgo, imperatore (I come re di Boemia) 286

Cassio Gaio Longino 289

Castellani Arrigo 320 n., 321, 345 n., 347, 351 n., 355 n.

Castracani Castruccio 323

Cataruza da Pesaro 164

Caterina d'Alessandria, santa 20

Catilina Lucio Sergio 248, 255, 258, 259 n., 260, 263-264, 274

Catone Marco Porcio, detto Uticense 274

Cavalca Domenico 11, 49 e n., 53, 55-56, 61, 63 e n., 65,191

Ceccano Annibaldo da 278, 281

Ceccano Giovanni II da 281

Ceccano Giovanni III da 281

Ceccano Goffredo da 281

Ceccano Margherita da 278 e n.

Ceccano Tomasio da 281

Ceccherini Irene 323

Ceffi Filippo 276 e n., 294 n., 300 n., 314, 340-341, 344 n., 345 n., 357, 359

Cella Roberta IX

Certeau Michel de $214 \mathrm{n}$.

Cesare Gaio Giulio 274 e n., 275, 276 e n., 277, 289-293

Cesario di Heisterbach $48 \mathrm{n}$.

Chiappa Miriam 357

Cicerone Marco Tullio X, 200, 248, 255
Cinico Giovan Marco 198

Cino da Pistoia 322

Ciociola Claudio $273 \mathrm{n}$.

Ciriaco, versificatore della Bibbia $94 \mathrm{n}$.

Claudio, imperatore 277

Codagnello Giovanni 244-246

Cola di Rienzo 285-286, 287 e n.

Colletta Pietro 175 e $n$.

Colombini Giovanni 88-89

Colombo Michele IX, $319 \mathrm{n}$.

Colonna Pietro 281

Colonna Sciarra 281

Colonna Stefano 281

Coluccia Rosario 215

Compagni Dino 47, 77, 211, 217, 225-226, 227 e n., 237

Conon de Béthune 311

Contini Gianfranco 23, 26, 35

Coppini Donatella 197

Cornagliotti Anna 86, $93 \mathrm{n}$.

Cornelio Nepote 332 e n., 333, 334 e n.

Costantino, imperatore 291 e n., 292 e n.

Cristoforo, santo 21

D’Achille Paolo, $215 \mathrm{n}$.

D’Acunto Nicolangelo VII

D’Agostino Alfonso $344 \mathrm{n}$.

Davanzati Bernardo 153, 347 e n., 352-353

Davanzati Bostico 352

De Blasi Nicola 338, 341, 345 n.

De Caprio Chiara IX, 215 n.

de’ Dazi Giovanni 21

De Luca Giuseppe 85-87

De Robertis Teresa 348

De Roberto Elisa VIII, 9, 22 e n.

de' Rossi Nicolò 165

de' Rossi Pino 199

de Rubeis Rainaldo 281

Del Balzo Angilberto 90 n., 91

del Bene Lanfranco $320 \mathrm{n}$.

del Grazia Soffredi 320 e n.

Delcorno Carlo 3,12

Della Bella Giano 241

della Scala Cangrande $330 \mathrm{n}$.

delle Colonne Guido XI, 275, 316, 319, 321-322, 329 e n., 332 n., 335 e n., 336 e n., 337 e n., 339, 344 n., 345 n., 346-347, 353-354, 359 n., 360 n. 
Dello Russo Michele 340

Diacciati Silvia 237 n., 239 n.

Divizia Paolo 200

Domenico di Guzmán, santo 13

Doni Anton Francesco 201

Druso Maggiore 277

Ducati Alice 319 n., 339 n.

Duccio di Gano 52

Elena madre di Costantino, santa $291 \mathrm{n}$., $292 \mathrm{n}$.

Eleonora d'Angiò, regina di Sicilia 49-50, 63

Elia da Cortona 15

Elia, profeta 74

Eliseo, profeta 74-75

Elliott Aaron Marshall 320

Enrico VII di Lussemburgo 286-287, 289, 292

Eusebio di Cesarea 220

Faini Enrico X, $268 \mathrm{n}$.

Federico II, imperatore 174, 176, 245

Federico III d'Aragona, re di Sicilia 50, 175, 193

Feo Michele 197

Ferdinando I d'Aragona, re di Napoli 198

Ferrari Mirella 155 n., 157

Ferri Simonetta $358 \mathrm{n}$.

Ficino Marsilio 200

Filelfo Francesco 198, 200

Filippo da Santa Croce 228, 261

Flacco Lucio Valerio 266

Folena Gianfranco 212-213

Formentin Vittorio 91 n., $155 \mathrm{n}$.

Franceschini Ezio 24-26, 37

Francesco I da Carrara, signore di Padova 197

Francesco d'Assisi 7-8, 11-14

Franco Niccolò 201

Froissart Jean 212

Fucci Vanni 328

Gasca Queirazza Giuliano 86

Gautier de Coincy 26, 41, 50 n.

Genette Gérard 222

Gerardo di Frachet 15

Gervasio di Tilbury 44

Gesù Cristo 151, 157-160, 162, 164
Ghazan, ilkhan di Persia 164 n.

Ghinazzone da Siena, detto Oriente Senese 93

Giacomino da Verona 150

Giacomo il Maggiore, santo 325

Giamboni Bono X, 211, 217 e n., 227, 228 e n., 237-238, 239 e n., 241 n., 242-244, 246-252, 261, 268 e n., 276

Gidino da Sommacampagna 150-151

Gioacchino da Fiore $188 \mathrm{n}$.

Giordano da Pisa 13, 15, 95 e n.

Giordano di Sgurgola 281

Giovanni dalle Celle 199

Giovanni da Parma 14

Giovanni di Salisbury 212

Giovanni Battista, santo 312-313

Girolami Remigio de' 250

Girolamo, santo 220, 275

Giulia, sorella di Gaio Giulio Cesare 277

Giuliari Giovan Battista Carlo 150-152

Giuseppe, figlio di Giacobbe 86

Giusti Maria 322 n.

Giusto, padre di Ormisda 283

Gobi Giovanni 312 n.

Goffredo da Bussero 21

Gonzalo de Berceo 26, 41, 50 n.

Gozzi Gozzo 87, 89

Gozzi Maria 339 n.

Gracco Gaio Sempronio, tribuno della plebe 247-249

Gracco Tiberio Sempronio, tribuno della plebe 247-249

Gregorio di Tours 47, 51

Gregorio Magno, santo VIII, 23 n., 46, 48 e n., 53 n., 54 e n., 56-57, 59, $60,62-63,65$ e n., $66,67,70,72-75$, $289 \mathrm{n}$.

Grévin Benoît $174 \mathrm{n}$.

Griffin Nathaniel Edward $321 \mathrm{n}$.

Grioni Franceschino $165 \mathrm{n}$.

Guadagnini Elisa $173 \mathrm{n}$.

Gualdo Riccardo $215 \mathrm{n}$.

Guenée Bernard 212-213

Guernes de Pont-Sainte-Maxence 20

Guevara Iñigo 93

Guglielmo da Volpiano 5

Guibert de Nogent 51

Guido da Pisa 273, 275-276, 284, 294 
Hugues Farsit 51

lacopo da Varazze 34-35

Ilarione di Gaza, santo 13

Innocenzo III, papa (Lotario dei Conti di

Segni) 6, 7 e $\mathrm{n}$.

Iorio-Fili Domenico $359 \mathrm{n}$.

Ippocrate di Coo 310

Isidoro di Siviglia, santo $221 \mathrm{n}$.

Jean de Haute-Seille 312

Jean le Conte $31 \mathrm{n}$.

Jenson Nicolas $92 \mathrm{n}$.

Jung Marc-René 336 n., 341

Koch Peter 166

Lami Giovanni $352 \mathrm{n}$.

Lancia Andrea 276, 300, 323

Latini Brunetto 173, 239, 256, 257 n., 259, 265 n., 268 e n., 269, 270

Laurent Françoise 20

Lavagna, Filippo Cavagni di 52

Lazzàri Rustichello de' 322 n.

Leca Marco 267

Leonardi Lino 86-87, 90

Lepido Manlio Emilio 266

Licht Tino 24

Livia Drusilla 277

Livio Tito 89, 198, 220-221, 228-229, 270, 285

Lorenzi Biondi Cristiano $273 \mathrm{n}$.

Lorenzi Cristiano X, 249 n., 355 n.

Lorenzo, padre di Cola di Rienzo 287

Lorenzo, santo 20

Lucano Marco Anneo 89, 220, 276 n.

Ludovico IV, imperatore, detto il Bavaro 323

Luigi IX, re di Francia, santo $186 \mathrm{n}$.

Luigi di Taranto, re titolare di Sicilia IX, 197-198

Macario il Grande, santo 15

Maggini Francesco 256-257

Malatesta Malatesta IV 334

Malerbi Nicolò VIII, 85, 91-92, 96

Manni Paola $322 \mathrm{n}$.

Manzi Adele 30

Marcello Marco 266
Marcello (pseudo Marcello) 191

Marco di Ghino da Prato 346

Margherita, santa 20-21

Maria di Francia 311

Maria Egiziaca, santa 21

Maria Maddalena, santa 151

Maria Vergine 25, 50, 51, 52 e n., 62, 70, 72, $75,76,160,161,327$

Mario Gaio 266

Marmochino Sante 92

Marogna Maria Antonietta 155 n.

Marsili Luigi 199

Martino IV, papa (Simon de Brion) 188

Martino V, papa (Ottone Colonna) 278

Martino Polono 220, 275-276

Masi Giuseppe 353

Massimiano, vescovo di Siracusa $48 \mathrm{n}$.

Massimo di Trevi 281

Matalena, madre di Cola di Rienzo 287

Matasci Joëlle 217

Mattia d'Anagni 281

Meiss Millard 10

Menichetti Caterina 86

Menocchio, $c f$. Scandella Domenico

Metello Quinto 266

Michele da Piazza $175 \mathrm{n}$.

Migliorini Bruno 153, $322 \mathrm{n}$.

Milani Giuliano 239 n.-240 n., 244

Mineo Ennio Igor 244-245

Mino da Colle 177

Minucio Rufo Marco 249

Monachi Ventura 302

Monti Carla Maria 201 e n.

Montuori Francesco $215 \mathrm{n}$.

Mordenti Raul $225 \mathrm{n}$.

Morf Heinrich 344 n., 357

Mussafia Adolfo IX, 150-153

Natale Sara 86-87, 90

Neckam Alessandro 312,

Neri Umberto $96 \mathrm{n}$.

Nerone, imperatore $184,188,191,193,275$, 276 n., 277, 285

Niccolò V, papa (Tomaso Parentucelli) $282 \mathrm{n}$.

Nicola di Nardò $90 \mathrm{n}$.

Nicolò di Lira $188 \mathrm{n}$.

Nigel di Canterbury 51 
Oddone di Cluny 5

Oesterreicher Wulf 166

Omero 224-225, 329, 331, 333-334

Omobono di Cremona 6

Oriente Senese, $c f$. Ghinazzone da Siena

Ormanni Agnolo 327

Ormisda, papa 283

Orosio Paolo X, 217, 220-222, 227-228, 237 e n., 238, 243-244, 246-248, 249 e n., 250-251, 261, 268, 276

Orsini Napoleone 285

Ottokar Nicola 248

Ovidio Nasone Publio 22 n., 89, 221 n., 300 n., 305, 329-330

Pagani Giuseppe Maria Gaetano 352

Paolo Diacono 276

Paolo, santo 12, 48, 191-192, $291 \mathrm{n}$.

Passavanti lacopo 90

Pellegrini Paolo VIII, 155 n., 319 n.

Petoletti Marco 202 n., 319 n., 330 n.

Petrarca Francesco IX, 197-201, 330 e n.

Pier Damiani 5, 12

Pier della Vigna 176

Pieri Paolino 230

Piero di Vaschino 341

Pietro, vescovo di Alessandria, santo 283

Pietro da Barsegapè $37 \mathrm{n}$.

Pietro da Napoli $94 \mathrm{n}$.

Pietro Comestore $23 \mathrm{n}$.

Pietro Leopoldo d'Asburgo-Lorena, granduca di Toscana 325

Pietro, santo 48, 158-159, 162, 164, 191, 283, $291 \mathrm{n}$.

Pilato Leonzio 329

Pipino Pietro 278

Plinio il Vecchio 198

Plutarco 198

Poggiali Gaetano 86 e n.

Polono Martino 220, 275-276

Pomaro Gabriella 155 n., 157, 319 n., 345 n., $351 \mathrm{n}$.

Pomian Krzysztof 232

Porcari Stefano 200

Pregnolato Simone XI, 225 n., 294 n.

Rainaldo di Supino 281
Rainuzzi Paolo 285

Ramello Laura 86

Ranieri da Pisa 6

Ranieri, vescovo di Volterra 177

Raterio di Verona 312

Rauty Natale $322 \mathrm{n}$.

Redi Francesco 87

Rehberg Andreas $273 \mathrm{n}$.

Revest Clémence $201 \mathrm{n}$.

Reynolds Leighton Durham 200

Rezzi Luigi Maria 256 e n.

Riccardi Gabriello 352

Ricci Ardingo 93

Ricci Gabriele 338

Ricci Romigi 93

Ricotta Veronica $273 \mathrm{n}$.

Roberto Grossatesta 14

Romualdo Salernitano $282 \mathrm{n}$.

Rossi Vittorio 197-198, 201 e n.

Russo Camilla $200 \mathrm{n}$.

Rustici Francesco 92

Sabatini Francesco $345 \mathrm{n}$.

Sacchetti Franco $360 \mathrm{n}$.

Salimbene de Adam 14-15

Salimbeni Benuccino de' 327

Sallustio Crispo Gaio 220, 248, 263, 332 n., $334 \mathrm{n}$.

Salomonio Ottaviano 52

Salutati Coluccio 334

Salvioni Carlo 149, 152-153

Sanzanome, giudice, autore dei Gesta Florentinorum 245 e n., 246

Savino Giancarlo 327 n., 355 n.

Scandella Domenico, detto Menocchio 294

Scarpini Cristina $94 \mathrm{n}$.

Schweickard Wolfgang $319 \mathrm{n}$.

Scipione Nasica Publio Cornelio 269

Segre Cesare 23 n., 212 n., 323 n., $359 \mathrm{n}$.

Seibt Gustav 226, 235

Semiramide, regina d'Assiria 250

Seneca Lucio Anneo 270

Sercambi Giovanni 230

Simintendi Arrigo 293

Simonetta Francesco, detto Cicco 52

Stefanini Ruggero $23 \mathrm{n}$. 
Stefano di Borbone 51

Stussi Alfredo 151-152

Svetonio Tranquillo Gaio 191, 228 n., 293

Syme Ronald 247 n., 248 n.

Taziano di Siria 149

Tedici Michele 327

Tommaseo Niccolò $345 \mathrm{n}$.

Tommaso d'Aquino 64

Tommaso da Celano 13

Tommaso di Eccleston 8, 14

Tommaso di Morolo 281

Tuttle Edwuard Fowler 153 e n.

Ugurgieri Andrea $333 \mathrm{n}$.

Vaccari Alberto 86

Vaccaro Giulio X, 217 n.

Valerio Massimo 89, 198, 261

Valerio Massimo Manio $247 \mathrm{n}$.

Vàrvaro Alberto 211 n., 212-213

Vauchez André 20, 21 e n.

Vegezio Renato Publio 237, 238 e n., 242-244, 250

Venanzio Fortunato, santo 47
Vesconti Jacopo 327

Vidmanová-Schmidtová, Anežka 23, 24 e n., 25 e n., 36

Villani Giovanni VIII, 47, 77-79, 174, 211, 218, 219 n., 221, 225-226, 227 n., 230, 282

Villani Matteo 217

Vincenzo di Beauvais 51, 220

Vindelino da Spira 91

Violante Cinzio 14

Virgilio Marone Publio 89, 198, 221 e n., 229, 330

Visconti Luchino 197

Vittore di Capua, santo 149

Volcacio Tullo Lucio 266

Wilhelm Raymund VIII, $155 \mathrm{n}$.

Zabbia Marino 220, 223 n.

Zaccagnini Guido 355 e n., 356, 358 e n.

Zaggia Massimo VIII, 155 n., 276 n.

Zambrini Francesco 150

Zinelli Fabio 86

Zorzi Andrea $240 \mathrm{n}$.

Zumthor Paul 155-156, 167 


\section{Indice dei manoscritti e dei documenti d'archivio}

ADMONT (STEIERMARK, AUSTRIA), STIFTSBIBLIOTHEK

Admont.

664: $29 \mathrm{n}$.

Bergamo, Biblioteca Civica Angelo Mai e ARCHIVI STORICI COMUNALI

MA 460: 157

MMB 808 (olim Gab. $\wedge$ I 20) $\left(=\right.$ Bgm $\left.^{2}\right)$ :

201-202, 204-207

BERLIN, StAATSBibLIOTHEK

Hamilton

67: 256 e n.

Bologna, Biblioteca Comunale DELL'ARCHIGINNASIO

A 252: $94 \mathrm{n}$.

CAMBRIDGE (MASSACHUSETTS), HARVARD UNIVERSITY, HOUGHTON LIBRARY

Riant

$87: 288 \mathrm{n}$

CAMBRIDGE (UK), UNIVERSITY LIBRARY

Additional

6685 (= AntTestCantabr): 85, 87, 89, 96 n., 97 e n., 100, 104, 108, 112, $116,120,124,128,132,136,140,144$

CATANiA, Biblioteche riunite Civica e A. URSINO RECUPERO

C 65: 273

CHICAGO, NEWBERRY LIBRARY

f 93: $288 \mathrm{n}$.

COPENAGHEN, Kongelige Bibliotek

Thott

128: 51

DURHAM, CATHEDRAL LIBRARY

C IV 24 (= D): 176, 181, 182 n. -187 n.
HARVARD, HaRvard College Library

Lat.

35: $321 \mathrm{n}$.

FIRENZE, ARCHIVIO dI STATO

Diplomatico

Pistoia, Comune (e S. Jacopo, Opere) Normali, 7 maggio 1282: $323 \mathrm{n}$.

Normali, 22 dicembre 1298: 323

Riccardi

Filza 237: 352

FiRENZE, Biblioteca Marucelliana

C 128: 256 e $n$.

C 140: $288 \mathrm{n}$.

firenZE, Biblioteca Medicea Laurenziana

Acquisti e Doni

399: $288 \mathrm{n}$.

424: 340

785: $94 \mathrm{n}$.

Ashburnham

1102 (= AntTestAshb): 87, 88 n., 96 n., 97 e n., 100, 104, 108, 112, 116, 120, 124, 128, 132, 136, 140, 144

Conventi Soppressi

122: 256 n. $-257 \mathrm{n}$.

Gaddi reliqui

18: $256 \mathrm{n}$.

35: 340

45: 340

71: X-XI, 299, 300 e n., 301, 304-305, 307 n., 308, 310, 312-313, 315, 316 e n. -317 e n., $338 \mathrm{n}$.

Medicei Palatini

115: $288 \mathrm{n}$.

153: 342

Plutei

43.21: $288 \mathrm{n}$.

61.22: $291 \mathrm{n}$.

61.26: $256 \mathrm{n}$.

89 inf. $-44: 340$

89 inf. $-56: 256$ n. 
91 sup. 52: $277 \mathrm{n}$.

91 sup. 53: $290 \mathrm{n}$.

Redi

113: $256 \mathrm{n}$.

Segni

4: $288 n$.

FIRENZE, BIBLIOTECA MORENIANA

Moreni

102: 352

FIRENZE, Biblioteca Nazionale Centrale

Banco Rari

8: 92 e $n$.

50: 202 e $n$.

Capponi

108: 282

Conventi soppressi

C 3.626 (= AntTestGozzi): 87, 96 n., 97 e n., 100, 104, 108, 112, 116, 120, 124, $128,132,136,140,144$

Fondo principale

II.I.19: $288 \mathrm{n}$.

II.I.363: $288 \mathrm{n}$.

II.I.93: $256 \mathrm{n}$.

II.II.23: 256 n. -257 n.

II.II.49: $291 \mathrm{n}$.

II.II.74: 277 n., 291 n.

II.II.87: 198

II.III.328: 256 n.

II.IV.32: $278 \mathrm{n}$.

II.IV.45: $333 \mathrm{n}$.

II.IV.46: 341

II.IV.107: $94 \mathrm{n}$.

II.IV.128: $256 \mathrm{n}$.

II.IV.279: $288 \mathrm{n}$.

II.IV.280: 289 n.

II.Iv.281: 289 n.

II.IX.27: 198

Magliabechiani

II.IV.49 (olim IV.49): 344 n.

XXIII.129: $289 \mathrm{n}$.

XXIII.136: $256 \mathrm{n}$.

XXXIV.1: $256 \mathrm{n}$.

XXXIX.49 (= MagIMiscColombini): 88, 95, 97 n., 98, 102, 106, 110, 114, 118, $122,126,130,134,138,142,146$
Nuovi Acquisti

1045: $94 \mathrm{n}$.

Palatini

1: $86 \mathrm{n}$.

126: $94 \mathrm{n}$.

459: $289 \mathrm{n}$.

471: $289 \mathrm{n}$.

502: 343

539: 256 e $n$.

568: 340

576: $94 \mathrm{n}$.

591: 282

674: $289 \mathrm{n}$

Panciatichiani

32: 313

41: $94 \mathrm{n}$.

51: $94 \mathrm{n}$.

53: $256 \mathrm{n}$.

55 (olim 88): 340

64: $289 \mathrm{n}$.

65: 291

FIRENZE, BIBLIOTECA RICCARDIANA

1095 (olim Gabriello II.III.380)

$\left(=R_{1}\right): 319,331$ e n., 332 n., 339 n., 340, 344 n., 345 e n.347 e n., 348-349, 354, 355 n., 356,357 e $n$.

1105: $256 \mathrm{n}$.

1252: $87 \mathrm{n}$.

1265: $94 \mathrm{n}$.

1279: $94 \mathrm{n}$.

1311: 341

1380: $94 \mathrm{n}$.

1513: $256 \mathrm{n}$.

1538: $256 \mathrm{n}$.

1550: $291 \mathrm{e}$.

1552: $256 \mathrm{n}$.

1553: $290 \mathrm{n}$.

1563: $256 \mathrm{n}$.

1628: $94 \mathrm{n}$.

1655: 93

1672: $94 \mathrm{n}$.

1821: 340

1900: 341

1954: 289 n.

2062: $289 \mathrm{n}$. 
2197 (= R): 175, 178, 181, 183-184, 186-187

2268 (= R2): 319, 321 n., 324, 331, 339 n., 340, 344 n., 345, 347 e n. -349 e n., 350, 351 n., 352, 354-356,

357 e n. -358 e n., 360 e $n$.

2418: $256 \mathrm{n}$.

2543: 355

3824: 352

4072: $256 \mathrm{n}$.

FULDA, HOCHSCHUL- UND LANDESBIBLIOTHEK 100 Bonifatianus 1: 149

KÓRNIK (POLONIA), PolsKa AKAdEMIA NAUK, BibLIOTEKA KóRNICKA

633: 256 n. -257 n.

LYON, BIBLIOTHÈQUE MUNICIPALE

1367: 87 n., 91

1368: 87 n., 91

LEIPZIG, UNIVERSITÄTSBIBLIOTHEK

1269: 201 n., 205

LONDON, BRITISH LIBRARY

Additional

15477: $321 \mathrm{n}$.

16437: 256 n. -257 n.

22155: $321 \mathrm{n}$.

36671: $321 \mathrm{n}$.

Egerton

1866: $289 \mathrm{n}$.

Harley

4123: $321 \mathrm{n}$.

3436: 202

Royal

15 C xvi: $321 \mathrm{n}$.

$20 \mathrm{DI}: 300 \mathrm{n}$.

LOS ANGELES, UNIVERSITY OF SOUTHERN

CALIFORNIA, HOOSE LIBRARY

091 A717e: 202

MANCHESTER, JOHN RYLANDS LIBRARY

Lat.

178 (olim 198): 202

\author{
MILANO, Archivio Storico Civico e \\ Biblioteca Trivulziana \\ Trivulziani \\ 92: 21 \\ 93: 21 \\ 545: $94 \mathrm{n}$. \\ milano, Biblioteca Ambrosiana \\ P 256 sup. $\left(=A m b r^{1}\right): 201,204-206$ \\ Q 36 sup.: 24
}

MOdENA, Biblioteca Estense e UNIVERSITARIA

Alpha G 5.10 (Ital. 382): $94 \mathrm{n}$.

Alpha H 3.6 (Ital. 437): 94 n.

milano, Biblioteca nazionale

BRaIDENSE

Morbio

$3(=M): 273,291$

MÜNCHEN, BAYERISCHE StAATSBIBLIOTHEK Clm 5350: 201 n., 205

NAPOLI, BibLIOTECA DELLA SOCIETÀ NAPOlETANA DI Storia PATRIA

XX C 5: 282

XXXII D 14 bis: 282

NAPOLI, Biblioteca Nazionale «VItTORIO EMANUELE III»

XIV D 7: 282

San Martino

I.63: 282

NEW HAVEN (CONNECTICUT), YALE UNIVERSity, BEINECKE LIBRARY

197: 202

NEW YORK, MORGAN LIBRARY \& MUSEUM

M 973: 217 n., 229-231, 282

OLOMOUC, StátNí OBLASTNí ARCHIV

C 0 418: 202

OXFord, Balliol College

Arch. E 2.4 (Coxe 126): 201 n., 204 
OXFORD, BODLEIAN LIBRARY

Canonici It.

94: $94 \mathrm{n}$.

126: $94 \mathrm{n}$.

133: 342

Laud. Misc.

503: 197, 202

PADOVA, Biblioteca Antoniana

47 scaff. II: 256 n., 342

PAlermo, Biblioteca Centrale della

Regione SiCILIANA

I D 14 (= P): 217 n., 230

PAlermo, Biblioteca Comunale

2 Qq E 1: 341

PALERMO, SOCIETÀ SICILIANA DI STORIA

PATRIA

IB 25: 176 e $n$.

PARIS, BIBLIOTHÈQUE DE L'UNIVERSITÉ 229 (perduto): 203

PARIS, BIBLIOTHÈQUE NATIONALE dE FRANCE Italiens

1 (= BibbiaNap): 85, 90-91, 93 n., 96, 97 e n., $98,101,105,109,113,117,121$, $125,129,133,137,141,145$

2: $90-91$

3: 91

4: 91

85: 93

109: $94 \mathrm{n}$.

120: 341

301: 282

303: 282

438: 273

$617(=\mathrm{G}): 341-342$

1095: $289 \mathrm{n}$.

Latins

2191: 198

5694: $321 \mathrm{n}$.

5695: $321 \mathrm{n}$.

Nouvelles acquisitions

503: $52 \mathrm{n}$.
PARma, Biblioteca Palatina

Palatini

313: $289 \mathrm{n}$.

Parmensi

1043: 341

PISA, ARChIVIO DI StATO

Alliata

65: 341

PISTOIA, ARChIVIO di StATO

Opera di San Jacopo

31: $328,348 \mathrm{n}$.

237: $319,324-325$

PISTOIA, Biblioteca Comunale FORTEGUERRIANA

A 53: 320

PRAHA, NÁROdNÍ KNIHOVNA

Roudnice

VI Fb 13 (Cim. J 342): 203

PRATO, ARCHIVIO DI STATO

Spedali

2607: 88

ROMA, ARCHIVIO DI STATO

522: $289 \mathrm{n}$.

ROMA, BiblioteCA ANGelicA

1552 (= AntTestAng): 85, 90, 91, 97 e n., 98, 101, 105, 109, 113, 117, 121, 125, 129, 133, 137, 141, 145

1553 (= AntTestAng): 90, 91, 97 e n., 98, $101,105,109,113,117,121,125,129$, 133, 137, 141, 145

1554: $94 \mathrm{n}$.

ROMA, Biblioteca Casanatense

D I.4: $289 \mathrm{n}$.

ROMA, Biblioteca dell'AcCADEMia NAZionale deI Lincei E Corsiniana

43 D 9 (1827): $94 \mathrm{n}$.

44 C 8 (olim Rossi 42) (=C): 173-178, 181-187, $256 \mathrm{n}$. 
44 D 24 (olim Rossi 43): 343

44 E 27: 289 n.

Siena, Biblioteca Comunale degli INTRONATI

F III 4 (= AntTestSen): 85, 88, 96 e n., 97 n., 98, 100, 104, 108, 112, $116,120,124,128,132,136$, 140, 144

I V 5 (= LibriStorATSen): 88, 97 n., 98, 102, 106, 110, 114, 118, 120, 124, $130,134,138,142,146$

IV 28: 49

UDINE, Biblioteca ARCiVescovile 108: 342

UPPSALA, UNIVERSITETSBIBLIOTEKET C 805: $94 \mathrm{n}$.

VATICANO (CITTÀ DEL), BIBLIOTECA Apostolica Vaticana

Barberiniani latini

3665: $94 \mathrm{n}$.

3953: $339 \mathrm{n}$.

4012: 198

4032: $52 \mathrm{n}$.

4045: $256 \mathrm{n}$.

LVI.56: 288 n., 289

Chigiani

L VI.230: 198

L VII.267 (= Ch $\left.^{2}\right)$ : 173-174, 176 e n., 177-182, 185-187, $256 \mathrm{n}$.

LVII.269: 198

M V.117: 288 n., 289, 290 n.

M VI.189: $288 \mathrm{n}$.
Latini

4601: 281 n., 282

4821: $94 \mathrm{n}$.

7208: $94 \mathrm{n}$.

Ottoboniani latini

1554: 201

2358: 203

Palatini latini

1596: 203

Vaticani latini

3355: 201

VEneZia, Biblioteca Nazionale Marciana

It.

II.13 (4937): $94 \mathrm{n}$.

VI.52 (6029): $94 \mathrm{n}$.

VI.81 (5975): 94 n., 343

VI.285 (6192): $94 \mathrm{n}$.

VIII.26 (6090): 256 n. -257 n.

XI.126 (6916): $289 \mathrm{n}$.

Z 13 (4744): 150

Z 18 (4793): $256 \mathrm{n}$.

Z 47 (4805): 342

Lat.

XIV.165 (4254): 199

Verona, Biblioteca Civica (olim Comunale) 753: $150 \mathrm{n}$.

WIEN, DOMINIKANERKLOSTER

$136\left(\right.$ Dom $\left.^{2}\right): 201$ n., 205

WIEN, ÖSterREICHISCHE NATIONALBIBLIOTHEK 3314: $94 \mathrm{n}$. 